\title{
AMPLITUDE DISTRIBUTION CONTROL OF ULTRASONIC VIBRATION TOOLS WITH A 2-D EXTENDED RADIATION SURFACE
}

\author{
Y.Koike", M.Kurosawa", S.Ueha", AND K.Adachi"*
}

"Precision and Intelligence Laboratory, Tokyo Institute of Technology, Yokohama 227 Japan

"Faculty of Engineering, Yamagata University, Yonezawa 992 Japan.

\begin{abstract}
The normal amplitude distribution of a 2 dimensionally extended radiation surface of an ultrasonic vibration tool is made uniform by a modal vibration control method which employs straight homs called 'Wave-Trapping Horns'. After numerically finding the tool shape which provides an in-phase longitudinal mode at the surface, the mode control is carried out. The displacement amplitude uniformity between the minimum and the maximum values along the surface is successfully increased from $75 \%$ to $85 \%$.
\end{abstract}

\section{Introduction}

Large ultrasonic vibration tools, which have a 2 dimensionally extended radiation surface, often require a uniform the normal amplitude distribution along the radiation surface. But it is difficult to design such tools if the dimension of the radiation surface is extended when compared to the longitudinal wave length. Due to the lack of a rational design method, a lots of trial constructions are necessary to get a desirable design. Some of the authors have proposed a modal vibration control method as a solution of such problems. ${ }^{[1]}$ In this method, straight horns called "Wave-Trapping Horn (WTH)" are used to control the amplitude distribution, and the wasteful processes can be greatly reduced. Using this method, whose validity has been proved to be valid for making the normal amplitude distribution of a 1 dimensionally extended radiation surface, we tried to control the amplitude distribution of the 2 dimensionally extended radiation surface of cuboidal tools with slots. As a result, the uniformity of the distribution, which is defined as a ratio of the minimum displacement amplitude to the maximum one on the surface, was successfully raised from $75 \%$ to $85 \%$.

\section{Brief description of the control method}

An example of the vibration tools under study is shown in Fig.1 together with "Wave-Trapping Homs (WTH)", and Fig.2 shows a generalized scheme of this vibration system. In this figure, $\left\{f_{d l}, f_{d a}, \ldots, f_{d n}\right\}$ are the forces necessary to control the amplitude distributions $\left\{\boldsymbol{u}_{s 1}, \boldsymbol{u}_{s 2}\right.$

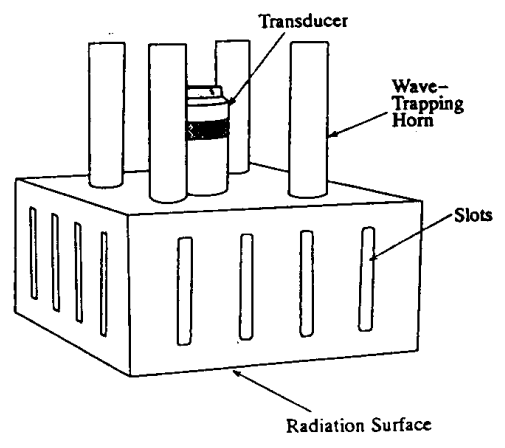

Fig.1 A modal vibration control system with use of "Wave-Trapping Horns (WTH)" for a tool with a 2dimensionally extended radiation surfaces.

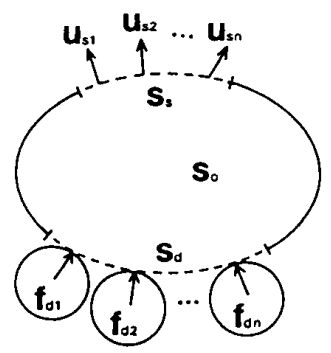

Fig.2 The schematic explanation of the vibration system.

,.., $\left.u_{s n}\right\}$, and are provided by the WTHs as in Fig.2. The letters $s, d$ and $o$ in the subscripts indicates the area where the values are defined, that is "the radiation surface", "the driving" and "the other" area, respectively. The numbers 1 through $n$ means discretising each area into $n$ elements.

These forces are calculated by the finite element method (FEM) using the equation of motion;

$$
-\omega^{2} M u+K u=f
$$

which is valid for a discretised linear, non-absorptive elastic system subjected to forced harmonic vibration. In this equation $\omega, M, K, u$ and $f$ are the angular frequency, the mass matrix, the stiffness matrix, the displacement and the driving force vectors, respectively. If we replace the matrix $-\omega^{2} M+K$ by the matrix $A$, the eq. 1 can be rewritten as: 


$$
\left(\begin{array}{lll}
A_{s s} & A_{s o} & A_{s d} \\
A_{s o} & A_{\infty} & A_{o d} \\
A_{s d} & A_{o d} & A_{d d}
\end{array}\right)\left(\begin{array}{l}
u_{s} \\
u_{o} \\
u_{d}
\end{array}\right)=\left(\begin{array}{l}
f_{s} \\
f_{0} \\
f_{d}
\end{array}\right)
$$

As $f_{d}=0$ and $f_{0}=0$ in this problem, we can calculate $f_{d}$ from this equation if the displacement $u_{s}$ and the driving frequency are given. The driving frequency is chosen nearby the resonance frequency of the in-phase longitudinal vibration, because the number of driving forces available is limited and a large perturbation of the amplitude distribution is impractical.

The demanded forces $f_{d}$ can be realized by attaching straight horns at the specified point as shown in Fig.2. The driving impedance $f_{d} / u_{d}$ at the specified point has to match with the impedance viewed from the driving end. Since the latter can vary from $-\infty$ to $+\infty$ depending on the length of the hom, as shown in Fig.3, the matching with the driving impedance is possible in any case. The length of the horn is calculated with the assumption that only a one-dimensional wave propagation is possible in the hom, and is written as follows:

$$
L=\frac{1}{k} \tan ^{-1}\left(\frac{f_{d}}{u_{d} k Y a}\right)+\frac{\pi}{k}
$$

where, $k=\omega \vee(Y / \rho), \rho$ the density, $Y$ the Young's modulus and $a$ the cross section of the hom, respectively. The original cuboidal part of the tool does not vibrate at the natural resonance frequency and is forced to vibrate near the resonance frequency with a specified amplitude distribution on the radiation surface. It should be noted that the forces yielded by a WTH are only effective to the normal displacement of the surface. Thus, the attached points have to be chosen where there is little in-plane displacement.

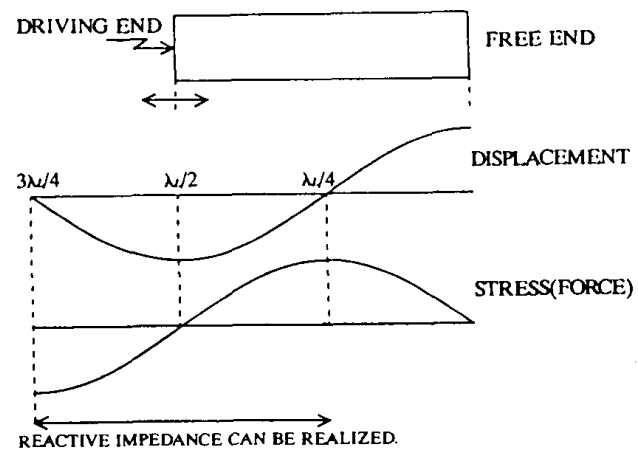

Fig. 3 The explanation of the realization of the forces using a straight horn in longitudinal mode.

\section{Modal vibration analysis of cuboidal vibrators}

The control method utilizing WTH requires that a tool has an in-phase longitudinal vibration mode at a given frequency, as mentioned before. To find such tools, the modal vibration analysis of various cuboidal vibrators with slots is carried out in advance by means of the finite element method (FEM). Figure 4 shows an example of a finite element representation of the cuboidal tool with slots. A tool, which has three slots along one direction and two slots along the other direction, may be called a " $3 \times 2$ model". The example in Fig. 4 is a " $2 \times 2$ model". The number of slots may be increased, depending on the dimension of the radiation surface. The parameters $\mathbf{W}, \mathbf{T}, \mathbf{S}$ and $\mathbf{H}$ denote "width of a column", "width of a bridge", "width of a slot" and "height of a tool", respectively. Since the amplitude uniformity of the radiation surface is the most important, the uniformity is calculated changing each dimension. Here, the amplitude uniformity is defined as a ratio the minimum amplitude to the maximum in the radiation plane. In this study, the dimension of parameters $\mathbf{W}, \mathbf{T}, \mathbf{S}, \mathbf{H}$ and the number of

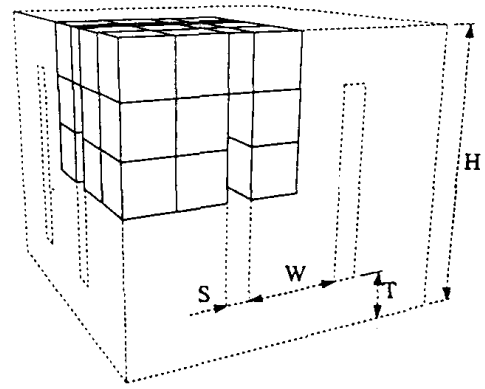

Fig.4 The finite element representation of the cuboidal tool of $2 \times 2$ model.
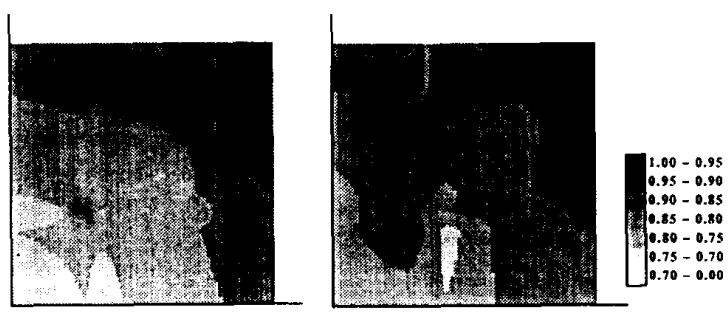

Fig.5 The comparison of the normal amplitude distribution between calculated result(Left) and experiment one(Right). 
slots are altered in each calculation. The number of lots was varied from two to four in one direction. Although the values $W, S$ and $T$ are simultaneously variable, the combination of these parameters was proven to have no influence to the in-phase longitudinal mode. And, $W$ and $S$ were also proven to have only slight effects on the mode. On the other hand, $T$ was proven to have much influence on the longitudinal mode. In keeping with the previous study ${ }^{[2]}, H, S, W$ are kept constant at $H=\lambda / 2$, $S=\lambda / 24, W=\lambda / 6 \quad(\lambda$ is the wave length of the longitudinal mode of a thin rod ) and only $T$ is chosen as a principal variable. The model is assumed to be made from Aluminum, whose constants are as follows: Young modulus $Y=6.85 \times 10^{10} \mathrm{~N} / \mathrm{m}^{2}$, Poisson's ratio $\sigma$ $=0.34$, density $\rho=2700 \mathrm{~kg} / \mathrm{m}^{3}$. Taking advantage of the symmetry of the desired longitudinal mode, one-eighth of the model is analyzed as shown in Fig.4. To confirm the accuracy of the analysis, the normal amplitude distribution at the radiation surface was measured for a trial-made $2 \times 2$ tool with $T=\lambda / 12$. Figure 5 shows the comparison between the experimental and the calculated results. In the figure, the normal displacement amplitude is normalized using the maximum amplitude and a quarter of the radiation surface is displayed. The darker density portion shows a higher amplitude. Both results show a good agreement with each other. The calculated resonance frequency $18.78 \mathrm{kHz}$ also agrees with the experimental one of $18.62 \mathrm{kHz}$. These results confirm the accuracy of the analysis. Figure 6a shows that the amplitude uniformity ratio against the parameter $\mathrm{T}$ for the models with a square radiation surface. Figure $6 \mathrm{~b}$ also shows that of the model with a rectangular radiation surface. The amplitude uniformity can obviously be improved by varying the value of $T$. As expected, a tool with a large radiation surface is found to vibrate less uniformly than that with a small one. This is because other than in the $2 \times 2$ model in the normal amplitude becomes smaller away from the center of the surface. The maximum value in this investigation, however, could not exceed $85.0 \%$ which is required for conventional ultrasonic plastic welding. Thus, some modal control method is required in order to achieve the demanded ratio.

\section{Simulation and experiment of the amplitude distribution control}

As pointed out in the previous section, even the dimensions of optimized tools need the amplitude distribution control. A $2 \times 2$ model was simulated to improve the uniformity distribution by this method.

Each dimension of the tool is $\mathrm{H}=\lambda / 2, W=\lambda / 6, S=\lambda / 24$ and $T=\lambda / 12$, respectively. The driving frequency and the position of the horns are given in advance. In order to find the driving frequency and the position which gives the maximum uniformity ratio, we have to vary these parameters by a small amount. Due to the geometrical limitation, the number of the hom is fixed at five throughout the experiment. As the amplitude distribution

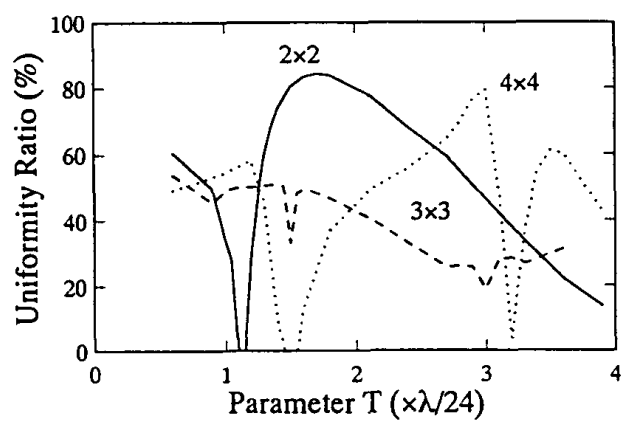

Fig.6a The variation of the amplitude uniformity ratio against the parameter $\mathbf{T}$ for the model with a square radiation surface.

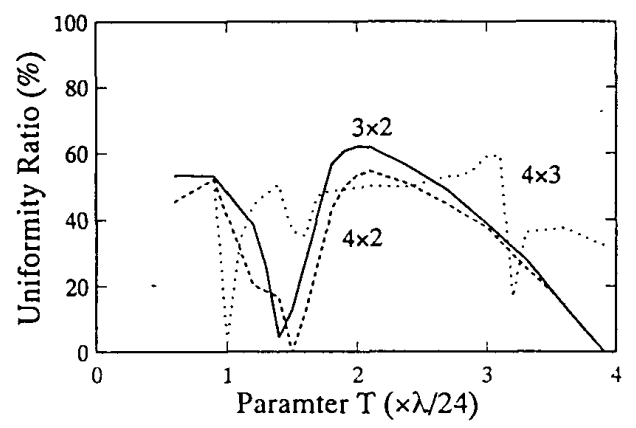

Fig.6b The variation of the amplitude uniformity ratio against the parameter $\mathbf{T}$ for the model with a rectangular radiation surface.

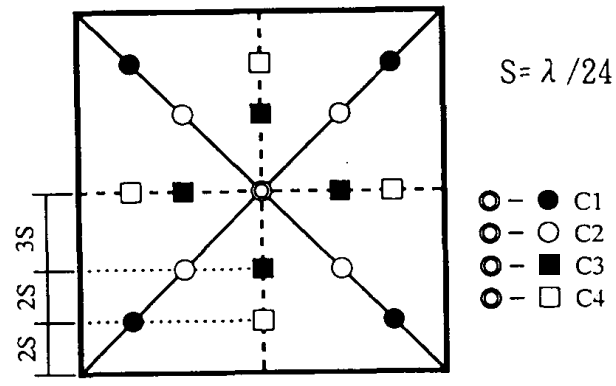

Fig.7 A schematic top view of the vibration tool of $2 \times 2$ model.

of an in-phase longitudinal mode is symmetric with respect to the center the arrangement of the horns should be symmetrical. Figure 7 shows a schematic top view of the vibration tool with five WTHs. Four arrangements $\mathrm{C}_{1}, \mathrm{C}_{2}, \mathrm{C}_{3}$ and $\mathrm{C}_{4}$ are examined. The junction points between the tool and the WTH correspond to the nodes in a FEM analysis model. As the amplitude distribution of an in-phase longitudinal mode is symmetric with respect to the center, it is appropriate that in this method the displacements of the horns are also symmetrical. The

1991 ULTRASONICS SYMPOSIUM - 985 
transducer was attached at the center in the experiment, and was treated as one of horns in the calculation. The driving frequency must be almost the same as the original resonance frequency for a forced vibration mode is to be analogous to a free longitudinal vibration mode. Figure 8 shows the calculated variation of the uniformity ratio against the frequency for the $2 \times 2$ model.Comparing the uniformity in the case of the bare cuboidal tool indicated by a dashed line, the control method clearly improves the amplitude uniformity of the radiation surface. In the experiment, modal vibration on the $2 \times 2$ model was controlled with use of the Aluminum WTH. The length $\mathrm{L}$ of each WTH is calculated in accordance with eq.(2). Since the junction point is assumed to be a point in the calculation, the contact surface is as narrow as possible. As the lateral vibration is easily to be transmitted to the horn due to the small contact area, the junction point is tapered. The WTHs are connected to the cuboidal tool with a screw made from steel. Figure 9 shows the variations of the WTHs' length and of the amplitude uniformity ratio at the radiation surface as a function of the driving frequency. The solid line indicates the calculated uniformity ratio in the calculation and the dotted line the calculated length of the horn. The experimental uniformity is plotted by the black dot and that of the length of the horn by the white dot. From this figure, the in-phase longitudinal vibration mode can obviously be realized by this modal control method. But there are some differences between the calculated and the experimental result at the high driving frequency. This may be due to the in-plane displacement at the attached positions.

Figure 10 shows the comparison of the normal amplitude distribution of the radiation surface at the condition indicated in Fig.9. The normal amplitudes are shown as in Fig.5 the same way in the previous. This also indicates that the measured result agrees to the calculated one.

\section{Conclusion}

The normal amplitude distribution of the cuboidal tool with a 2 dimensionally extended radiation surface is controlled by the modal control method which utilizes wave trapping horns. The uniformity ratio on a $2 \times 2$ model was successfully improved from $75 \%$ from $85 \%$ in the experiment.

\section{Reference}

[1] K.Adachi and S.Ueha,"Modal vibration control of large ultrasonic tools with the use of wave-trapped homs", in "JAcout.Soc.Am.87(1),January 1990",p.208 [2] K.Adachi,S.Ueha, and E.Mori,"Modal Vibration Analysis of Ultrasonic Plastic Welding Tools Using the Finite Element Method," in The Proceedings of Ultrasonics International 85"(Butterworths, London, 1986),p.727.

986 - 1991 ULTRASONICS SYMPOSIUM

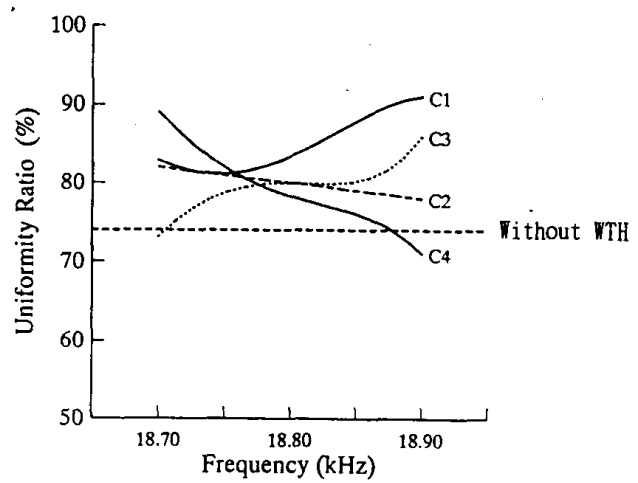

Fig.8 The variation of the uniformity ratio against the frequency for $2 \times 2$ model

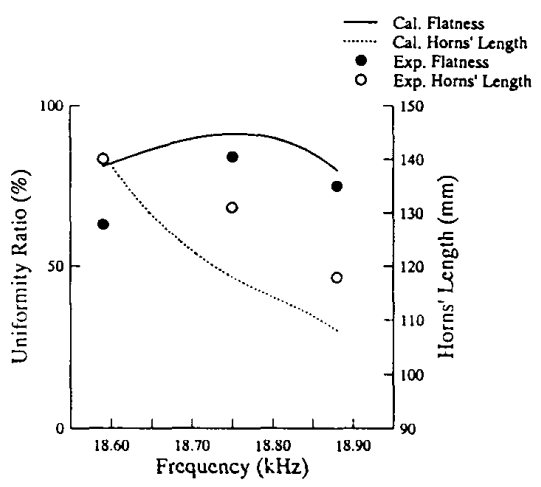

Fig.9 The variation of the wave-trapped horns' length and of the amplitude uniformity ratio at the radiation surface as a function of the driving frequency.
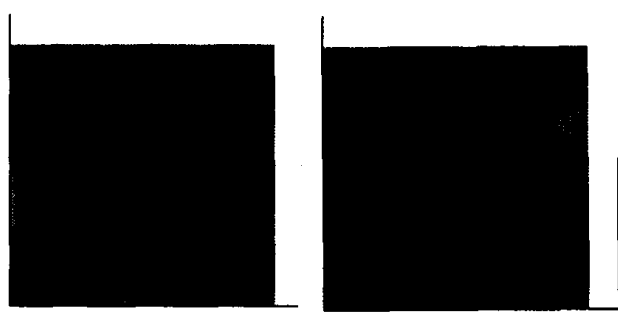

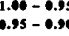

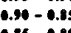

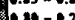

Fig.10 The comparison of the normal amplitude distribution of the radiation surface between calculated result(left) and the experimental one(right) at the condition indicated in Fig.9. 